\title{
A possible basis for conservatism in signal detection and probabilistic categorization tasks
}

\author{
MICHAEL KUBOVY \\ Yale University, New Haven, Connecticut 06520
}

\begin{abstract}
It is commonly claimed that conservative placement of the criterion in signal detection is due to the form of the utility function of money, to conservatism in the estimation of prior probabilities, or to probability matching tendencies. This article shows how conservatism could be caused by a systematic misconception of the shape of the underlying distributions. An experiment is described in which subjects were asked to make posterior probability judgments after performing numerical analogues of signal detection. The posterior probability judgments were radical, i.e., high posterior probabilities were overestimated and low posterior probabilities were underestimated; if this pattern of radical probability estimation reflects the subjects' understanding of the underlying distributions, it would account for conservative criterion placement.
\end{abstract}

Green and Swets (1966/1974, p. 91) discuss two sets of signal detection data bearing on the relation between the obtained criterion $(\beta)$ and the optimal criterion $\left(\beta^{*}\right)$ : The first set were collected by Tanner, Swets, and Green (Note 1) from a single participant in an auditory experiment, who was induced to shift $\beta$ by varying the a priori probabilities of signal occurrence. The second set of data were collected by Green and Swets from the same person, who was at that time induced to shift $\beta$ by varying the values of the decision outcomes. This participant did not use $\beta^{*}$ : when $\beta^{*}>1, \beta$ tended to be smaller than $\beta^{*}$, and when $\beta^{*}<1, \beta$ tended to be larger than $\beta^{*}$. Such behavior is said to be conservative, because it manifests the decision maker's tendency to avoid extreme criteria.

A number of explanations have been proposed for this conservative behavior. (1) Green and Swets maintain that since "the experimenter's principal interest is in a sensory process ... [the subject] probably finds it difficult to believe that he would be performing resonsibly if the sensory distinctions he makes are exactly those that he would make by removing the earphones in an auditory experiment or

The data in this article come from the author's dissertation (Kubovy, 1971). The data collection was supported by grants from the Faculty of Social Sciences of the Hebrew University of Jerusalem and the Marshall Fund for Research in Social Psychology. Later phases of this work were supported by PHS Grant MH 26573 from the National Institutes of Mental Health, and by a Junior Faculty Fellowship to the author from Yale University. The author gratefully acknowledges the encouragement and help of his dissertation advisor, Professor A. Tversky. Reprints may be requested from Michael Kubovy, Department of Psychology, Yale University, Yale Station Box 11A, New Haven, Connecticut 06520. by turning his back on a visual signal"' (p. 92). (2) Galanter (1974) and Ulehla (1966) suggest that conservatism with varying values of the decision outcomes could be accounted for by the form of the utility function of money. (3) Analogously, Ulehla (1966) argues that conservatism with varying a priori probabilities could be accounted for by the underestimation of high a priori probabilities, and the overestimation of low a priori probabilities. (4) A number of investigators have proposed that subjects tend to match the probability of their "yes" responses to the probability of signal presentation (Creelman \& Donaldson, 1968; Dorfman, 1969; Parks, 1966; Thomas \& Lesse, 1970), which could account for conservatism. [However, as Thomas (1975) points out, the probability matching hypothesis has not fared well: Subjects are generally more conservative than probability matching predicts.]

The four possibilities just enumerated all assume that the source of conservatism is in the responsebiasing process. A fifth possibility must be added to them, which would locate the source of conservatism in a misconception of the form of the stimulus distributions or a misconception of the relationships between the stimulus distributions.

Suppose, for example, that observers underestimate the height of the tails of the normal distribution. In such a case, likelihood ratios greater than 1 would be overestimated, and likelihood ratios that are smaller than 1 would be underestimated. Equivalently, since the posterior probability of an observation is a strictly monotone function of the likelihood ratio (Egan, 1975, pp. 12-13), posterior probabilities greater than 0.5 would be overestimated, and posterior probabilities smaller than 0.5 would be underestimated. We will follow 
Hammerton (1973) in saying of such a pattern of probability estimation that it is radical.

To see how radical posterior probability judgment relates to conservative criterion placement, consider an observer who is ideal in all respects but one: his or her knowledge of the distributions is deficient. Suppose that such a deficient observer were placed in a signal detection situation with an a priori probability of signal plus noise of 0.25 , and a symmetric payoff matrix. Since the calculation of $\beta^{*}$ does not require any knowledge of the distributions, our deficient observer will do so correctly, choosing a criterion $\beta^{*}=3$. Because the posterior probability of observation $\mathrm{x}$ is equal to $1(\mathrm{x}) /[1+1(\mathrm{x})]$, where $1(x)$ is the likelihood ratio of $x$, the criterion $\beta^{*}=3$ corresponds to a posterior probability of 0.75 . Suppose, furthermore, that the observer's deficiency is one of radical posterior probability judgment. Then what will be judged by the deficient ideal observer to be a criterion $\beta^{*}=3$ will in fact be less extreme, say $\beta=2.5$ (corresponding to a posterior probability of 0.71 ), and the resultant criterion placement will appear to be conservative.

Any data about criterion placement can be interpreted either as a result of criterion selection or in terms of the knowledge of underlying distributions. A convenient way to interpret data in terms of knowledge of the distributions is to plot the posterior probability corresponding to the ideal $\beta^{*}$ as a function of the posterior probability corresponding to the obtained $\beta$. Such a plot purposely reverses independent and dependent variables in order to emphasize that if only the subject's knowledge of the distributions is distorted, the point on the decision axis corresponding to the subject's criterion $\beta$ would be judged by the subject to correspond to $\beta^{*}$.

In Figure 1, we present such a plot of the data reported by Green and Swets $(1966 / 1974$, p. 91) and discussed in the beginning of this paper. The figure shows very clearly the pattern of judgments that could underlie the placement of the criterion. Both conditions ("values variable," corresponding to a manipulation of $\beta$ by varying payoffs, and "probabilities variable," corresponding to a manipulation of $\beta$ by varying a priori probabilities) show a pattern of radical probability judgment. We note a discrepancy, however, between the two conditions: The values-variable condition produces the greatest degree of conservatism, and hence implies more radical posterior probability judgments. If the data for the two conditions had been collected from the same observer at approximately the same time, his misconception of the distributions would be expected to be the same in both conditions. In such a case, a discrepancy would suggest that the hypothesis about the misconception of the distributions is incorrect, or

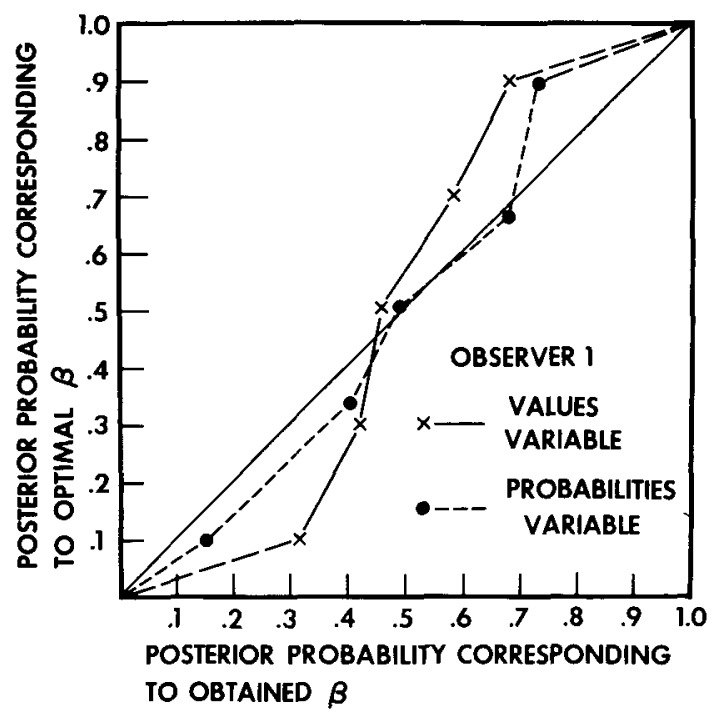

Figure 1. Data replotted from Green and Swets $(1966 / 1974)$ to show that conservatism in criterion placement is equivalent to radicalism in posterior probability estimation.

at least that some additional factors counteract the effect of radical judgment in the probabilities-variable condition. We do not know, however, when the data were collected from this observer, and therefore need not speculate further on the source of the discrepancy.

Figure 2 shows the same type of plot for data obtained by Kubovy, Rapoport, and Tversky (1971) in a numerical decision task in which six subjects were shown four-digit numbers said to represent the heights of men and women in millimeters, and were asked to decide whether each height belonged to a

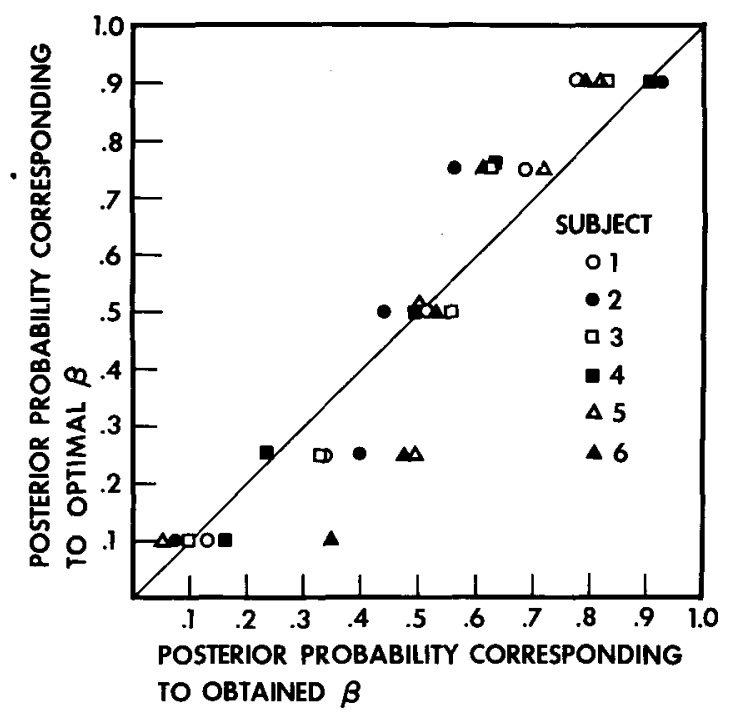

Figure 2. Data replotted from Kubovy et al. (1971) to illustrate the same point as Figure 1. 
man or to a woman. Since in these data, as in the data of Green and Swets, the subjects tended to place their criteria conservatively, the inferred pattern of posterior probability judgments is also radical.

This article reports data relevant to the distribution misconception hypothesis. Subjects participating in a study of decision biases caused by interpersonal influence (Kubovy, 1971) were run in a design with alternating noninfluence and influence trials. Although certain types of bias were manifested by subjects on influence trials, none of the influence exerted was of a nature to induce radical probability estimation on either influence or noninfluence trials. The Method section below covers the entire procedure of the experiment in some detail. The data of interest, however, were drawn from only part of the experiment in question: the noninfluence trials in sessions during which subjects were asked for their posterior probability judgments. The reason for reporting Method in such detail is to allow the reader to form an opinion on whether the data may have been contaminated, with respect to the hypothesis in question, by their having been collected as baseline data in an experiment designed with a different purpose in mind.

\section{METHOD}

\section{Subjects}

The subjects were 12 volunteers-10 females and 2 malesfrom the 10th and 11 th grades (sophomores and juniors) in three high schools in Jerusalem, Israel.

\section{Procedure}

The subjects were divided into two groups of six. Each group was run in a large room, facing a screen on which the stimuli were projected at a rate of approximately $6 / \mathrm{min}$. For each group, 12 sessions were conducted; each session lasted between 1 and $2 \mathrm{~h}$ (including a 10-min break halfway through the session). The sessions were scattered over a period of a month. Four special sessions were conducted for subjects who could not attend regular sessions.

\section{Stimuli}

The stimuli were four-digit numbers. The numbers were sampled at random from two distributions (denoted $a$ and b) whose means were $m(a)=1,630$ and $m(b)=1,797$, and standard deviations $s(a)=s(b)=167\left(d^{\prime}=1\right)$. Tables of the standard normal density function were used to sample 500 numbers so as to provide the best approximation to distribution a. A further sample of 500 numbers from distribution $b$ was prepared by adding 167 to the numbers sampled from a. The 1,000 numbers thus obtained were printed in duplicate on 2 by 2 in. transparencies to form a pool of 2,000 slides.

\section{Structure of the Sessions}

Each session consisted of 400 trials, on each of which one transparency was projected. There were two types of sessions with respect to the nature of the subjects' task.

Binary response sessions (Sessions 1-5). Subjects were asked to decide on each trial whether the stimulus had been sampled from $\mathrm{a}$ or $\mathrm{b}$, and to indicate their decision by writing $\mathrm{A}$ or $\mathrm{B}$ on a response sheet. Subjects were told that the distributions $a$ and $b$ represented the heights of women and men, respectively, but that they need not resemble any adult population they were familiar with; that the distributions were unimodal, symmetric, and partially overlapping; and that men and women had equal chances of being sampled on each trial. The subjects were presented on each trial with a single stimulus drawn randomly and with equal probability from a or $b$. Correct binary feedback was provided on even-numbered trials (called feedback trials; see below).

Posterior probability estimation sessions (Sessions 6-12). The subjects were asked to estimate on each trial the probability that the stimulus had been sampled from $b$, and to indicate their estimates by writing a number between 0 and 100 on a response sheet. The subjects were told to imagine on each trial a room containing 100 people whose heights were given by the stimulus number, and to estimate how many of them would be men.

Each session consisted of an alternating series of two types of trials:

Influence trials (odd-numbered trials, except in Sessions 1 and 6). On these trials, the experimenter informed the subjects of the responses of a fictive "earlier participant" after projecting the stimulus and before the subjects recorded their responses. During binary response sessions, the influence responses were binary; during posterior probability estimation sessions, the influence responses took the form of posterior probability estimates.

Influence trials will not be analyzed in the present article, which is exclusively concerned with the subjects' posterior probability estimates in the absence of social influence, i.e., on the feedback trials in Sessions 7-12, which are discussed below. The nature of the information given on influence trials needs to be clarified, because it will be argued below that whatever response biases we observe in the feedback trials were not caused by the influence provided on the influence trials.

The influence responses during binary response sessions were selected as follows: For observation $x$, the probability that the influence response was $\mathrm{B}$ equaled the probability that $\mathrm{x}$ had been drawn from $b: p(B \mid x)=p(b \mid x)$. No bias was involved in these influence responses, since $p(A \mid x)=p(a \mid x)$.

The influence responses during the first four posterior probability estimation sessions (Sessions 7-10, if we exclude Session 6, a practice session) were selected as follows: For each observation $\mathrm{x}$, an observation $\mathrm{y}$ was drawn from the same distribution. If the difference in posterior probability of $y$ and $x$ did not exceed a predetermined value, $d$, the number $100 \times \mathrm{p}(\mathrm{b} \mid \mathrm{y})$, rounded to an integer, was used as inflinence response. Otherwise, y was sampled again. For one group of subjects (Group 1), $d=.35$ on Sessions 7 and 10 and $d=.20$ on Sessions 8 and 9 . For the other (Group 2), $d=.20$ on Sessions 7 and 10 and $d=.35$ on Sessions 8 and 9. No bias was involved: The percentage of influence responses which constituted overestimates was between 49 and 51 .

During the last two posterior probability estimation sessions (Sessions 11 and 12), the influence responses were selected differently for the two groups of subjects. Let $p=p(b \mid x)$ and let $s$ be the influence response. For Group $1, \mathrm{~s}=2.8 \mathrm{p} /[(1-\mathrm{p})+$ $2.8 \mathrm{pl}$; for Group $2, \mathrm{~s}=\mathrm{p}^{266} /\left[(1-\mathrm{p})^{266}+\mathrm{p}^{266}\right]$. It is easy to see that the influence responses received by Group 1 represent systematic overestimates of the posterior probability of $b$, $p(b \mid x)$. The influence responses given Group 2 represent overestimates of posterior probabilities less than 0.5 , and underestimates of posterior probabilities greater than 0.5. Although biases were introduced on the influence trials of these two sessions (Sessions 11 and 12), they were not instances of radical probability estimation. It will therefore be assumed that if the influence stimuli had any effect on the subjects' responses on the feedback trial, it would tend to obscure any pattern of radical probability estimation.

Feedback trials (even-numbered trials). On these trials, the subjects recorded their responses immediately after seeing the 
stimuli. Binary feedback was provided throughout the experiment. At no stage of the experiment was any numeric feedback provided that could effect the subjects' posterior probability estimates.

\section{RESULTS}

Figure 3 shows the results obtained on the feedback trials of Sessions 8, 9 (top panel), 7, 10 (center panel), and 11,12 (bottom panel), which were the probability estimation sessions, for Subjects 9 and 10.

The results for 9 of the 12 subjects show the same distinctive pattern: an S-shaped curve, manifesting an underestimation of low probabilities and an overestimation of high probabilities. The 3 subjects who do not appear to conform to this pattern of radical probability estimations are not, however, conservative. They all appear to overestimate the probability that a given height represents a man.

The subjects whose estimates are radical tend to overestimate the heights of men more than they underestimate the heights of women (see Figure 3).

Subjects appear to be extremely consistent from one session to another, suggesting that there is a nonarbitrary conceptualization underlying their probability judgments that can be said to constitute an individual "signature."

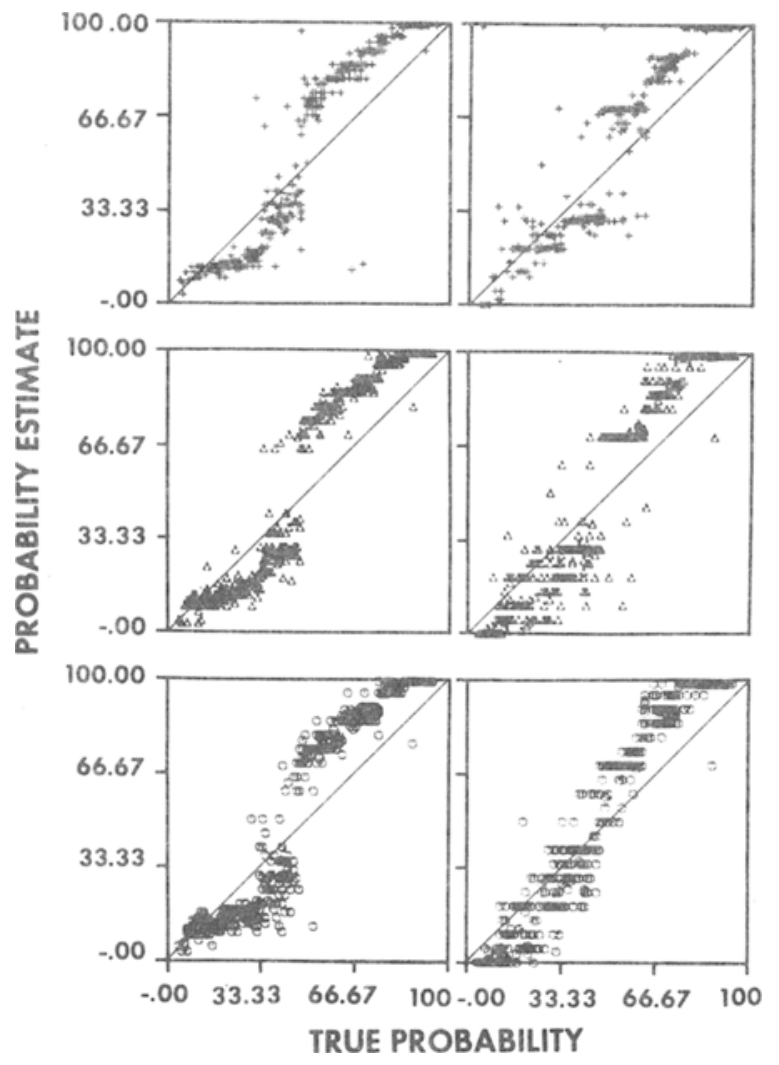

Figure 3. Posterior probability estimates as a function of true posterior probability for Subjects 9 and 10.
The data for each subject were grouped into three sets of 400 responses: (1) Sessions 7 and 10; (2) Sessions 8 and 9; (3) Sessions 11 and 12.

For each data set of each subject, I performed a stepwise polynomial regression of probability estimate $(\pi)$ on true probability (p). The order of the polynomial for each data set was the highest order that significantly incremented the variance accounted for by all lower order polynomials. Let us denote by $\pi^{\prime}$ the probability estimate predicted by the polynomial regression equation.

In order to test whether the subjects' probability estimates were radical, two measures of radicalism were devised, using the plots of the polynomial regressions. If a subject is radical, we should expect that $\pi^{\prime}>p$ for high values of $p$ and $\pi^{\prime}<p$ for low values of $p$. This is the basis for the first test of radicalism I performed. Assume that if $\pi^{\prime}=p$ for one and only one value of $p$, then at that value of $p$ the slope of the polynomial function $\pi^{\prime}=f(p)$ should be greater than unity if the subject is radical. Unfortunately, in some cases $\pi^{\prime}=p$ for more than one value of $p$. It was therefore decided to define radicalism (for our present purposes) in terms of the number of times each regression line crosses the line $\pi=p$ (i.e., the positive diagonal) with a slope greater than 1 (positive crossings), and the number of times it crosses the positive diagonal with a slope less than 1 (negative crossings). For 5 of the 12 subjects, the regression curves were nonmonotonic with $p$, but only for quite extreme values of $p$, thus crossing the positive diagonal with a negative slope, and hence spuriously crossing the positive diagonal with a slope less than 1. These crossings were ignored in the analysis. Table 1 shows the number of regression lines for each subject favoring radicalism by this criterion, and the number favoring conservatism (11 of 36 curves favored neither). The results are overwhelmingly in favor of radicalism (sign test: $p<$ $10^{-5}$ ).

In order to strengthen this conclusion in a way that cannot be affected by nonmonotonicity in the regression lines, the slope of each regression line at its point of intersection with the line, $\pi=1-p$ (the negative diagonal) was measured. If the slope of the regression line at that point is greater than 1 , it is an indication of radicalism. Table 1 shows the number of regression lines for each subject favoring radicalism, and the number favoring conservatism (5 of 36 curves favored neither). These results are overwhelmingly in favor of radicalism (sign test: $\left.\mathrm{p}<2 \times 10^{-5}\right)$.

\section{DISCUSSION}

The results have strongly confirmed our conjecture that subjects may be making their decisions under a radical illusion. The shapes of the probability estimation curves are quite similar to those presented in 
Table 1

Number of Curves Per Subject Favoring Radicalism and Conservatism, Using Two Different Criteria

\begin{tabular}{|c|c|c|c|c|}
\hline \multirow[b]{3}{*}{ Subject } & \multicolumn{4}{|c|}{ Diagonal Criterion } \\
\hline & \multicolumn{2}{|c|}{ Positive } & \multicolumn{2}{|c|}{ Negative } \\
\hline & $\begin{array}{l}\text { Radi- } \\
\text { calism }\end{array}$ & $\begin{array}{l}\text { Conser- } \\
\text { vatism }\end{array}$ & $\begin{array}{l}\text { Radi- } \\
\text { calism }\end{array}$ & $\begin{array}{c}\text { Conser- } \\
\text { vatism }\end{array}$ \\
\hline 1 & 2 & 0 & 3 & 0 \\
\hline 2 & 3 & 0 & 3 & 0 \\
\hline 3 & 1 & 0 & 3 & 0 \\
\hline 4 & 2 & 0 & 3 & 0 \\
\hline 5 & 3 & 0 & 3 & 0 \\
\hline 6 & 3 & 0 & 3 & 0 \\
\hline 7 & 3 & 0 & 0 & 1 \\
\hline 8 & 0 & 1 & 0 & 2 \\
\hline 9 & 0 & 0 & 3 & 0 \\
\hline 10 & 3 & 0 & 3 & 0 \\
\hline 11 & $i$ & 1 & 1 & 1 \\
\hline 12 & 2 & 0 & 3 & 0 \\
\hline Total & 23 & 2 & 28 & 4 \\
\hline
\end{tabular}

Figures 1 and 2, and must therefore be considered as candidates for the explanation of the phenomenon of conservatism in the location of the criterion in probabilistic categorization tasks, including signal detection.

These results should not, however, be taken as proof that radical probability judgments underlie the conservative criterion placements. First, they were not obtained concurrently with criterion placements, and could be unrelated to the criterion placements. Second, even if it were established that the subjective probabilities that determine criterion placements are radical, it would not demonstrate that they are sufficiently radical to account for the degree of conservatism observed in probabilistic categorization experiments. In order to provide us with information about the source of the phenomenon of conservatism in these tasks, we must devise procedures in which the various potential sources are isolated. Specifically, probabilistic categorization problems can be thought of as presenting two separate problems for the subjects. First, the subject must know how to use prior probabilities or the payoff matrix in order to place the criterion in an optimal location. Second, the subject must be able to assimilate information about the distributions, and in particular about the tails of the distributions, where very few observations are given. Experiments are underway to disentangle these two problems in order to assess the contribution of each to the phenomenon of conservatism.
Finally, the present results suggest that the terms "conservatism" and "radicalism" must be used with caution. For, as we have seen, radical probability estimation could be the cause of conservative criterion placement. Thus subjects should not be said to be radical or conservative in complex tasks such as cascaded decision tasks (Peterson, 1973) in which the output of one decision serves as input to another, or in tasks requiring the integration of multiple judgments. Each subtask can induce radical or conservative behavior. Only careful analysis can predict whether the final outcome of a set of decisions will appear to be radical or conservative.

\section{REFERENCE NOTE}

1. Tanner, W. P., Jr., Swets, J. A., \& Green, D. M. Some general properties of the hearing mechanism. Ann Arbor, Mich: University of Michigan Electronic Defense Group (Technical Report No. 30), 1956.

\section{REFERENCES}

Creelman. C. D., \& Donaldson, W. ROC curves for discrimination of linear extent. Joumal of Experimental Psychology, 1968, 77, 514-516.

Dorfman, D. D. Probability matching in signal detection. Psychonomic Science, 1969, 17, 103.

EgAN, J. P. Signal detection theory and ROC analysis. New York: Academic Press, 1975.

Galanter, E. Psychological decision mechanisms and perception. In E. C. Carterette \& M. P. Friedman (Eds.), Handbook of perception (Vol. 2). New York: Academic Press, 1974.

GreEN, D. M., \& Swets, J. A. Signal detection theory and psychophysics. Huntington, N.Y: Krieger, 1974 (originally published, 1966).

Hammerton, M. A case of radical probability estimation. Journal of Experimental Psychology, 1973, 101, 252-254.

Kubovy, M. Normative and informational aspects of social influence.] Unpublished doctoral dissertation, Hebrew University of Jerusalem, Israel, 1971.

Kubovy, M., Rapoport, A., \& TVersky, A. Deterministic vs. probabilistic strategies in detection. Perception \& Psychophysics, 1971, 9. 427-429.

PARKs, T. E. Signal-detectability theory of recognition memory performance. Psychological Review, 1966. 73, 44-58.

Peterson, C. R. (Ed.) Special issue: Cascaded inference. Organizational Behavior \& Human Performance, 1973, 10. $315-432$

Thomas. E. A. C. Criterion adjustment and probability matching. Perception \& Psychophysics, 1975, 18, 158-162.

Thomas, E. A. C., \& Legge, D. Probability matching as a basis for detection and recognition decisions. Psychological Review. $1970,77,65.72$.

UlehlA, Z. J. Optimality of perceptual decision criteria. Journal of Experimental Psychology, 1966, 71, 564-569.

(Received for publication February 3, 1977; revision accepted May 20, 1977.) 